\title{
Review Article \\ Haploidentical Hematopoietic Stem Cell Transplantation: Expanding the Horizon for Hematologic Disorders
}

\author{
Mohammad Faizan Zahid ${ }^{1}$ and David Alan Rizzieri ${ }^{2}$ \\ ${ }^{1}$ Aga Khan University, Karachi 74800, Pakistan \\ ${ }^{2}$ Division of Hematologic Malignancies and Cellular Therapy, Duke Cancer Institute, Durham, NC 27710, USA \\ Correspondence should be addressed to Mohammad Faizan Zahid; faizanzahid91@hotmail.com
}

Received 10 November 2015; Accepted 10 January 2016

Academic Editor: Suparno Chakrabarti

Copyright (c) 2016 M. F. Zahid and D. A. Rizzieri. This is an open access article distributed under the Creative Commons Attribution License, which permits unrestricted use, distribution, and reproduction in any medium, provided the original work is properly cited.

\begin{abstract}
Despite the advent of targeted therapies and novel agents, allogeneic hematopoietic stem cell transplantation remains the only curative modality in the management of hematologic disorders. The necessity to find an HLA-matched related donor is a major obstacle that compromises the widespread application and development of this field. Matched unrelated donors and umbilical cord blood have emerged as alternative sources of donor stem cells; however, the cost of maintaining donor registries and cord blood banks is very high and even impractical in developing countries. Almost every patient has an HLA haploidentical relative in the family, meaning that haploidentical donors are potential sources of stem cells, especially in situations where cord blood or matched unrelated donors are not easily available. Due to the high rates of graft failure and graft-versus-host disease, haploidentical transplant was not considered a feasible option up until the late 20th century, when strategies such as "megadose stem cell infusions" and posttransplantation immunosuppression with cyclophosphamide showed the ability to overcome the HLA disparity barrier and significantly improve the rates of engraftment and reduce the incidence and severity of graft-versus-host disease. Newer technologies of graft manipulation have also yielded the same effects in addition to preserving the antileukemic cells in the donor graft.
\end{abstract}

Today's age represents a truly unprecedented time in care of the patient with a hematologic malignancy with the advent of several targeted therapies and novel agents. These therapies have shown tremendous efficacy in treating several malignancies, yielding impressive response rates with limited toxicity profiles [1]. Despite substantial activity against different cancer types, relapse is often encountered at some point during the clinical course of most treated patients, with durable remissions being observed in only a certain percentage of patients. Allogeneic hematopoietic stem cell transplantation (allo-HSCT) is often the only treatment modality which can offer a cure to not only malignant but also benign hematological disorders [2]. A human leukocyte antigen- (HLA-) matched sibling donor is the preferred source of a stem cell graft in allo-HSCT. However, only $30 \%$ of patients requiring an allo-HSCT will have an HLA-matched sibling donor [3]. For patients not having a matched sibling donor, a matched unrelated donor (MUD) is an alternate source of stem cells, the probability of finding which is up to $75 \%$ among Caucasians. However, these chances are much lower among non-Caucasian populations, with $40 \%$ chance in Hispanic individuals and less than 20\% among Asian and African American individuals [3-5]. In addition, the cost of recruiting MUDs and maintaining donor registries is a major drawback, making this approach unaffordable and even impractical for developing countries. Hence, it is important to explore other possible stem cell sources.

Unrelated umbilical cord blood (UCB) represents another alternate source of stem cells, one which has been proven to be successful when performing allo-HSCT [6-10]. Key advantages include a comparatively quick donor search and a shorter time to proceed to transplant when compared with adult MUDs [11, 12]. This is especially important in the context of advanced/high-risk hematologic malignancies, where the risk of disease relapse is high and the goal is to proceed to a transplant while the patient is still in remission 
and has minimal disease burden after induction therapy [13]. In addition, UCB transplantation allows for a greater degree of HLA mismatch, most likely due to a lower number of activated alloreactive $\mathrm{T}$ lymphocytes present in cord blood $[14,15]$. This accounts for very low and acceptable rates of graft-versus-host disease (GvHD) following UCB transplants $[8,12,16]$. One of the obstacles to UCB transplants is the nonavailability of a sufficient number of hematopoietic progenitor cells in an UCB unit [12]. Although this limitation can be overcome by using more than one unit of cord blood, it is daunted by the high cost of maintaining cord blood banks [17]. Delayed engraftment, increased time to hematopoietic recovery, and immune reconstitution lead to substantially increased risks of life-threatening infections and graft failure, often offsetting the advantages of using unrelated UCB as a stem cell source $[5,12]$. Furthermore, due to a limited number of hematopoietic progenitor cells, posttransplant immune manipulation, such as donor lymphocyte infusions, is very difficult, which severely limits treatment options for patients who relapse after transplant $[6,12]$.

On the other hand, haploidentical related donors offer several key advantages over MUDs and UCB. Almost all patients have at least one haploidentical related donor in the family and the best donor amongst all candidate family members can be selected. Haploidentical related donors are readily available and are highly motivated to donate to a family member $[18,19]$. Due to rapid availability of donors and substantially lower costs, patients can proceed to transplant fairly quickly (as early as 3 weeks) [18]. Another major advantage of haploidentical donors over UCB is easy access to the stem cell source that makes donor-derived cellular therapy, such as donor lymphocyte infusions and immune manipulation, available for use after transplant, if necessary [18].

The HLA mismatch between the haploidentical donor and the recipient offers a potent graft-versus-tumor (GvT) effect to completely eradicate malignant cells and offer a permanent cure [18]. Some studies have reported substantially reduced relapse rates with greater degree of HLA mismatches [20-22]. However, two immunological barriers need to be overcome: (1) graft rejection, or host-versus-graft effect, and (2) GvHD. In allo-HSCT studies involving myeloablative conditioning (MAC) regimens, HLA mismatches, either at the antigen level or at allele level, have been associated with inferior survival and poor outcomes after allo-HSCT, with greater degree of mismatch correlating with worse outcomes $[21,23,24]$. Since donor-recipient HLA histocompatibility is the most important independent predictor of outcomes after allo-HSCT, these two barriers are more difficult to overcome in haploidentical hematopoietic stem cell transplantation (haplo-HSCT) than in HLA-matched allo-HSCT. Although the lower risk of relapse due to HLA disparity supports the existence of a GvT effect, this positive aspect of haplo-HSCT is offset by markedly increased rates of GvHD, graft failure, and nonrelapse mortality $[23,25]$.

Several attempts to perform haplo-HSCT using T lymphocyte replete, unmanipulated grafts have been made. Since donor-derived T lymphocytes are the fundamental players in the pathogenesis of GvHD, these attempts have been associated with early and severe multiorgan failure and GvHD, leading to alarming rates of transplant-related mortality despite pharmacological GvHD prophylaxis [26]. To reduce the risk of GvHD, a new approach of highly purified CD34+ stem cell grafts was adopted using ex vivo T lymphocyte depletion technologies [27]. With this strategy, almost no GvHD was observed in the recipients, despite the absence of posttransplant immunosuppressants for GvHD prophylaxis [28-30]. However, the small number of T lymphocytes transplanted using the highly purified CD34+ stem cell strategy does not allow for an efficient transfer of adoptive immunity, resulting in substantially delayed immune reconstitution and frequent, often fatal, infectious complications [18, 31]. Also, the risk of graft failure is also higher with $\mathrm{T}$ lymphocyte depleted haplo-HSCT [27, 32].

There is a key difference between positive selection of CD34+ progenitor cells and negative depletion of lymphocytes during graft manipulation. With CD34+ selection strategies, almost no cells other than the CD34+ stem cells are transplanted. On the other hand, negative depletion techniques (mainly CD3+ and CD19+ negative depletion) allows for other types of cells, such as dendritic cells, natural-killer (NK) cells, and monocytes, to be retained in the donor graft and transplanted with the CD34+ progenitors. Both graft manipulation techniques result in effective removal of $\mathrm{B}$ and T lymphocytes, leading to greatly reduced risks of developing posttransplant Epstein-Barr virus related lymphoproliferative disorders (B lymphocyte depletion) and GvHD (T lymphocyte depletion) $[26,33]$. The detailed research and broad application of graft manipulation has provided insight into the biology of NK cells, lymphocytic constituents of the innate immune system, and their immunotherapeutic potential in allo- and haplo-HSCT [34].

NK cell activity is modulated by the interaction between killer cell immunoglobulin-like receptor (KIR) expressed on the surface of NK cells and cognate ligands (i.e., certain HLA alleles) to KIRs [35]. Engagement of KIRs by their corresponding ligands produces inhibitory signals and prevents NK cell activation. In the context of allo-HSCT, and especially haplo-HSCT, NK cells in the allograft can attack malignant cells in the recipient if there is a mismatch between the KIRs on the donor-derived NK cells and the HLA antigens on the recipient (and malignant) cells [36]. With so many haploidentical donors available for a vast majority of patients, the ideal donor can be picked based on the KIR haplotype on the donor NK cells to exploit their alloreactivity and induce powerful GvT effects [37, 38]. Additionally, the disparity between KIRs and HLA haplotypes does not increase the risk of developing GvHD [39].

Another fundamental difference between CD34+ selection and negative depletion of lymphocytes (CD3+/CD19+ depletion) is that the number of alloreactive lymphocytes "contaminating" the donor graft is approximately ten times higher in $\mathrm{CD} 3+/ \mathrm{CD} 19+$ depletion. This necessitates the use of pharmacological GvHD prophylaxis after transplants using CD3+/CD19+ depleted grafts [33, 40]. However, a notable problem with the transplantation of T lymphocyte depleted grafts is the increased likelihood of relapse after transplantation $[41,42]$. A more efficient approach is the depletion of T-cell receptor (TCR) $\alpha \beta+\mathrm{T}$ lymphocytes and CD19+ cells 
(TCR $\alpha \beta+/ C D 19+$ depletion) from donor grafts $[43,44]$. This approach greatly increases the efficiency of alloreactive $\mathrm{T}$ lymphocyte removal (similar to that of CD34+ selection techniques), while retaining other cells such as NK cells and monocytes. In addition, this method also retains $\gamma \delta+\mathrm{T}$ lymphocytes in the graft. $\gamma \delta+$ T lymphocytes constitute approximately $5 \%$ of circulating T lymphocytes. These cells are nonalloreactive and exhibit potent antitumor and anti-infectious properties $[5,44]$. Since these cells are not activated by major histocompatibility complex (MHC) interactions, they do not react to alloantigens and hence do not contribute to GvHD $[45,46]$. This is advocated by the fact that patients exhibiting high $\gamma \delta+$ T lymphocyte counts after allo-HSCT show better survival with reduced incidence of relapse and GvHD [47]. In contrast, the majority of circulating T lymphocytes (approximately 95\%) are $\alpha \beta+\mathrm{T}$ lymphocytes which act against alloantigens via $\mathrm{MHC}$ interactions and give rise to GvHD after allo-HSCT [48]. The TCR $\alpha \beta+/ C D 19+$ depletion technique allows for satisfactory removal of $\alpha \beta+$ T lymphocytes to prevent GvHD while retaining $\gamma \delta+\mathrm{T}$ lymphocytes to ensure timely immune reconstitution and a robust GvT effect, especially after haplo-HSCT $[47,49,50]$. The most recent study [51] reporting the results of haplo-HSCT with TCR $\alpha \beta+/ C D 19+$ depletion in pediatric patients showed acceptable rates of GvHD (22\%). In all but one patient, GvHD was grade 2 and responded to first-line therapy. Graft failure was observed in $27 \%$ of patients, all of whom were successfully retransplanted with different rescue protocols. The overall survival of patients from this study was $96.7 \%$, advocating the efficacy of TCR $\alpha \beta+/ C D 19+$ depletion in improving outcomes after haplo-HSCT.

During the 1980s, outcomes of haplo-HSCT were discouraging due to severe GvHD and graft failure, often approaching rates as high as $90 \%[52,53]$. The introduction of graft manipulation and $\mathrm{T}$ lymphocyte depletion brought about notable improvements in outcomes of recipients of haploHSCT by dramatically reducing the incidence of GvHD [54]; however, the high incidence of graft failure remained a major obstacle to the application of haplo-HSCT [55, 56]. Graft failure is mediated by native cytotoxic $\mathrm{T}$ lymphocytes that persist in the recipient's system even after conditioning regimens are administered [57]. This barrier in histocompatibility was shown to be overcome in several animal-model studies [58] and, subsequently, in clinical studies $[57,59,60]$ by infusing high doses of $\mathrm{T}$ lymphocyte depleted hematopoietic progenitor cells. Additional strategies to help engraftment included enhancing the ablative intensity of conditioning regimens and addition of anti-T lymphocyte agents [57, 59, 61]. Unfortunately, such intensive conditioning regimens result in extensive organ damage and toxicities which limit their use to younger and relatively healthier patients in comparison to individuals of older age groups [5]. Chemotherapeutic doses and total body irradiation used in MAC regimens cause extensive damage to host tissue and release of inflammatory cytokines, such as tumor necrosis factor alpha $(\mathrm{TNF} \alpha)$ and interferon gamma (IFN $\gamma)$. These inflammatory mediators, and several others, play an integral role in the pathophysiology of GvHD [62], which may be one of the reasons why MAC regimen transplants are frequently complicated by
GvHD [57, 63]. The problem with T lymphocyte depleted grafts is delayed immune reconstitution and significant period of immunodeficiency which predisposes to serious and often fatal infections $[28,57,64]$.

Nonmyeloablative conditioning (NMAC) regimens are an attractive alternative for patients requiring an allo-HSCT who are not considered suitable candidates for MAC regimens due to advanced age, comorbidities, and/or increased risks of therapy-related adverse effects [65]. Using NMAC regimens also results in the decreased release of inflammatory mediators and cytokines and may consequently reduce the incidence and magnitude of GvHD, especially when used for haplo-HSCT. This significantly broadens the applicability of transplantation to patients who would otherwise be unfit to receive a transplant using standard protocols. Earlier studies applying haplo-HSCT with NMAC regimens showed discouraging patient outcomes, especially with alarming rates of graft failure [66, 67]. Rizzieri et al. [68] published the first large study on haplo-HSCT with fludarabine, cyclophosphamide, and alemtuzumab used as the preparatory NMAC regimen which demonstrated that the complications of GvHD and graft failure after haplo-HSCT could be overcome, making it a feasible treatment option in the therapy of patients with hematologic malignancies. $75 \%$ of the patients in this study achieved complete remission with only $10.2 \%$ transplant-related mortality in the first 100 days. Engraftment rate was high, with only $14 \%$ of patients suffering from graft failure, while only $8 \%$ of patients developed GvHD. However, relapse and infectious complications were major causes of mortality in this study ( $49 \%$ and $22 \%$, resp.). One plausible reason for the high rate of relapse could be that the patients participating in this study harbored poor-risk hematologic malignancies which carry an inherent probability of relapse.

Cyclophosphamide is a highly immunosuppressive alkylating agent with an established role in anticancer chemotherapy and HSCT [69]. Historically, cyclophosphamide is commonly administered as part of several conditioning regimens prior to HSCT; however, as previously discussed, the cytotoxic effects of this agent lead to tissue damage and release of inflammatory mediators and increase the risk of GvHD [62]. Administration of cyclophosphamide between 48 and 72 hours after transplant has been shown to facilitate posttransplant immune-tolerance; however, this tolerance is not induced when the drug is administered at 24 or 96 hours after transplant [70]. Cyclophosphamide exploits the high cytotoxic sensitivity of alloreactive T lymphocytes (both host and donor) to DNA damage [71]; hence carefully timed administration of posttransplant cyclophosphamide inhibits GvHD and graft rejection [72, 73]. O'Donnell et al. [69] investigated the role of posttransplantation cyclophosphamide in patients receiving haplo-HSCT after NMAC conditioning. The results of their study concluded that this strategy substantially reduced the risks of GvHD and graft rejection and was able to produce long-lasting donor-recipient chimerism after transplant. Subsequently, Luznik et al. [64] reported their experience with using posttransplant cyclophosphamide after NMAC haplo-HSCT. The results of their study showed acceptable rates of graft failure (13\%) and GvHD (grades 2-4: $34 \%$, grades 3-4: 6\%) with rapid achievement of 
donor-recipient chimerism after transplant. Administration of two doses of cyclophosphamide in the 48-to-72-hour window was associated with a lower risk of chronic GvHD than only one dose, further advocating that posttransplant cyclophosphamide is efficacious in inducing posttransplant tolerance. However, relapse of primary disease was a major cause of treatment failure and mortality (up to 51\%). This study also recruited patients with poor-risk hematologic malignancies, which may explain the high incidence of relapse in this study group. Patients with lymphoid malignancies were at a lower risk of experiencing relapse than those with myeloid malignancies, indicating particular effectiveness of cyclophosphamide in treating lymphoid hematologic malignancies. Other studies have demonstrated similar findings, corroborating that this strategy depletes alloreactive $\mathrm{T}$ lymphocytes in the donor graft as well as the host immune system, dramatically reducing the risk of both GvHD and graft failure, which are much more difficult to control in the setting of haplo-HSCT and are substantial impediments [69]. While there is concern that posttransplant cyclophosphamide might damage or kill donor hematopoietic progenitors cells, these cells have high expression of aldehyde dehydrogenase enzymes which confer relative resistance to the cytotoxic effects of the drug [74].

A relatively newer strategy to increase the success rates of haplo-HSCT is the use of T regulatory cells (Tregs). Tregs are a specialized subset of CD4+ lymphocytes with concurrent expression of CD25 and FOXP3 genes which play crucial roles in antitumor immunity, posttransplant tolerance, and protection against autoimmunity [75-77]. In addition, they have low expression of CD127 [78]. The adoptive transfer of these specialized T lymphocytes limits graft and host alloreactivity, leading to reduced risks of GvHD and graft failure [79-82]. Tregs also preserve and maintain normal lymph node and thymus architecture from GvHD and allow for a quick posttransplant immune recovery while having no negative effects on the GvT responses of allo-HSCT [83]. Additional studies have also showed that Tregs become activated and proliferate by interaction with recipient antigen-presenting cells in the proinflammatory milieu after administration of conditioning regimens, attenuating alloreactive $\mathrm{T}$ lymphocyte activation while allowing nonalloreactive $\mathrm{T}$ lymphocyte expansion to ensure a timely immune recovery $[82,84-86]$.

Di Ianni et al. [87] performed the first human study evaluating the adoptive transfer of Tregs on posttransplantation outcomes in patients receiving haplo-HSCT. Early infusion of donor-derived Tregs on day -4 of transplant, followed by infusion of positively immunoselected CD34+ stem cells and conventional $\mathrm{T}$ lymphocytes on day 0 , showed prevention of GvHD in the absence of any immunosuppressive GvHD prophylaxis and hastened posttransplant immune recovery and immunity against opportunistic pathogens and did not have any negative effects on GvT effects [87]. A follow-up study of these patients [84] showed successful engraftment in $26 / 28$ (93\%) patients. Only 2 (7.7\%) of 26 evaluable patients developed grade 2-4 GvHD. Spectratyping analysis showed the rapid development of $\mathrm{T}$ lymphocyte repertoire within months after transplant. Naïve and memory $\mathrm{T}$ lymphocyte subpopulations showed rapid increase over the first year after transplant, while CD4+ and CD8+ lymphocytes against opportunistic pathogens like Aspergillus and Toxoplasma appeared significantly earlier in comparison to historical controls comprising standard haplo-HSCT recipients. At a median follow-up of 21 months, $46.1 \%$ were alive and in remission [84].

Tregs can be isolated in vitro using standard donorderived leukapheresis products using double negative selection with anti-CD8 and anti-CD19 monoclonal antibodies and positive selection for CD25 [88]. Expression of the FOXP3 gene represents the peripheral mature Tregs [89], while low CD127 expression correlates with exhibition of regulatory functions in this $\mathrm{T}$ lymphocyte subset [90]. Such cell-based transplantation and graft modifications prove to improve posttransplantation engraftment, allow quick immune reconstitution, and reduce GvHD; however, the expertise required for such cellular manipulations limits their clinical implementation to highly specialized medical centers.

With regard to Tregs, posttransplant GvHD prophylaxis may also play a role in selectively favoring the expansion of Tregs while suppressing mature effector $\mathrm{T}$ lymphocytes. Sirolimus is an immunosuppressant that demonstrates such properties [91]. Peccatori et al. [92] performed a recent study showing that a calcineurin inhibitor-free, sirolimusbased GvHD prophylaxis regimen promoted selective Treg expansion after haplo-HSCT. This strategy was well tolerated and a robust and rapid engraftment was observed in the majority of patients. While the incidence and severity of acute and chronic GvHD in this study were comparable to historical data from MUD allo-HSCT using peripheral blood stem cells $[93,94]$, they were much higher when compared with haplo-HSCT using bone marrow as the source of donor stem cells $[64,95,96]$. In addition, Tregs show a higher level of resistance to the cytotoxic effects of cyclophosphamide [97]. Future studies may be performed to ascertain the specific effects of GvHD prophylaxis in T lymphocyte dynamics to systematize the utility of regimens based on their effects on $\mathrm{T}$ lymphocyte dynamics to derive the maximum benefit, carefully weighing GvHD, GvT, engraftment, and immune recovery after transplant.

In conclusion, allo-HSCT remains the only treatment modality that offers a potential cure for some with hematologic disorders. HLA-matched donors are not always available, which has led to establishment of UCB and MUDs as alternative sources for stem cell transplantation. However, the high cost of maintaining donor registries and cord blood banks, limited size of the cord blood units, and high-risk nature of the ablative procedure limit the expansion of these donor sources as well. HLA haploidentical donors are an attractive choice for stem cells since nearly every patient has an available haploidentical donor from their family. In modern medicine, new strategies such as "megadose stem cell infusions" and posttransplantation immunosuppression with cyclophosphamide have shown the ability to overcome graft failure and GvHD, complications that occurred at overwhelmingly high rates and severely limited the application of haploidentical transplantation before the 21st century. Other strategies such as NMAC regimens prior to transplant and pretransplant graft manipulation have demonstrated similar 
survival rates when compared with HLA-matched allo-HSCT with MAC regimens, but with significantly reduced toxicities associated with HSCT and preservation of the antileukemic properties of immune cells in the donor graft. Future prospective studies to explore the biology of the GvT effects in this setting can provide more perspective on the advantages and drawbacks of haplo-HSCT over "traditional" allo-HSCT, suggest further enhancements of the process, and move this "now-controversial" modality into the standard of care for patients with limited options.

\section{Conflict of Interests}

The authors declare that there is no conflict of interests regarding the publication of this paper.

\section{References}

[1] S. Ciavarella, A. Milano, F. Dammacco, and F. Silvestris, "Targeted therapies in cancer," BioDrugs, vol. 24, no. 2, pp. 77-88, 2010.

[2] E. A. Copelan, "Hematopoietic stem-cell transplantation," The New England Journal of Medicine, vol. 354, no. 17, pp. 1813-1826, 2006.

[3] L. Gragert, M. Eapen, E. Williams et al., "HLA match likelihoods for hematopoietic stem-cell grafts in the U.S. registry," The New England Journal of Medicine, vol. 371, no. 4, pp. 339348, 2014.

[4] J. Dehn, M. Arora, S. Spellman et al., "Unrelated donor hematopoietic cell transplantation: factors associated with a better HLA match," Biology of Blood and Marrow Transplantation, vol. 14, no. 12, pp. 1334-1340, 2008.

[5] M. Shabbir-Moosajee, L. Lombardi, and S. O. Ciurea, "An overview of conditioning regimens for haploidentical stem cell transplantation with post-transplantation cyclophosphamide," American Journal of Hematology, vol. 90, no. 6, pp. 541-548, 2015.

[6] K. K. Ballen, E. Gluckman, and H. E. Broxmeyer, "Umbilical cord blood transplantation: the first 25 years and beyond," Blood, vol. 122, no. 4, pp. 491-498, 2013.

[7] N. J. Chao, L.-P. Koh, G. D. Long et al., "Adult recipients of umbilical cord blood transplants after nonmyeloablative preparative regimens," Biology of Blood and Marrow Transplantation, vol. 10, no. 8, pp. 569-575, 2004.

[8] M. J. Laughlin, J. Barker, B. Bambach et al., "Hematopoietic engraftment and survival in adult recipients of umbilical-cord blood from unrelated donors," The New England Journal of Medicine, vol. 344, no. 24, pp. 1815-1822, 2001.

[9] G. D. Long, M. Laughlin, B. Madan et al., "Unrelated umbilical cord blood transplantation in adult patients," Biology of Blood and Marrow Transplantation, vol. 9, no. 12, pp. 772-780, 2003.

[10] D. A. Rizzieri, G. D. Long, J. J. Vredenburgh et al., "Successful allogeneic engraftment of mismatched unrelated cord blood following a nonmyeloablative preparative regimen," Blood, vol. 98, no. 12, pp. 3486-3488, 2001.

[11] J. N. Barker, T. P. Krepski, T. E. DeFor, S. M. Davies, J. E. Wagner, and D. J. Weisdorf, "Searching for unrelated donor hematopoietic stem cells: availability and speed of umbilical cord blood versus bone marrow," Biology of Blood and Marrow Transplantation, vol. 8, no. 5, pp. 257-260, 2002.
[12] J. Munoz, N. Shah, K. Rezvani et al., "Concise review: umbilical cord blood transplantation: past, present, and future," Stem Cells Translational Medicine, vol. 3, no. 12, pp. 1435-1443, 2014.

[13] U. D. Bayraktar, R. E. Champlin, and S. O. Ciurea, "Progress in haploidentical stem cell transplantation," Biology of Blood and Marrow Transplantation, vol. 18, no. 3, pp. 372-380, 2012.

[14] L. Garderet, N. Dulphy, C. Douay et al., "The umbilical cord blood $\alpha \beta$ T-cell repertoire: characteristics of a polyclonal and naive but completely formed repertoire," Blood, vol. 91, no. 1, pp. 340-346, 1998.

[15] E. W. Petersdorf, T. A. Gooley, C. Anasetti et al., "Optimizing outcome after unrelated marrow transplantation by comprehensive matching of HLA class I and II alleles in the donor and recipient," Blood, vol. 92, no. 10, pp. 3515-3520, 1998.

[16] J. E. Wagner, J. Rosenthal, R. Sweetman et al., "Successful transplantation of HLA-matched and HLA-mismatched umbilical cord blood from unrelated donors: analysis of engraftment and acute graft- versus-host disease," Blood, vol. 88, no. 3, pp. 795802, 1996.

[17] T. Bart, M. Boo, S. Balabanova et al., "Impact of selection of cord blood units from the United States and Swiss registries on the cost of banking operations," Transfusion Medicine and Hemotherapy, vol. 40, no. 1, pp. 14-20, 2013.

[18] Y. Reisner, F. Aversa, and M. F. Martelli, "Haploidentical hematopoietic stem cell transplantation: state of art," Bone Marrow Transplantation, vol. 50, supplement 2, pp. S1-S5, 2015.

[19] J. A. Roth, M. E. Bensink, P. V. O’Donnell, E. J. Fuchs, M. Eapen, and S. D. Ramsey, "Design of a cost-effectiveness analysis alongside a randomized trial of transplantation using umbilical cord blood versus HLA-haploidentical related bone marrow in advanced hematologic cancer," Journal of Comparative Effectiveness Research, vol. 3, no. 2, pp. 135-144, 2014.

[20] C. Anasetti, P. G. Beatty, R. Storb et al., "Effect of HLA incompatibility on graft-versus-host disease, relapse, and survival after marrow transplantation for patients with leukemia or lymphoma," Human Immunology, vol. 29, no. 2, pp. 79-91, 1990.

[21] R. C. Ash, M. M. Horowitz, R. P. Gale et al., "Bone marrow transplantation from related donors other than HLA-identical siblings: effect of T cell depletion," Bone Marrow Transplantation, vol. 7, no. 6, pp. 443-452, 1991.

[22] Y. Kanda, S. Chiba, H. Hirai et al., "Allogeneic hematopoietic stem cell transplantation from family members other than HLA-identical siblings over the last decade (1991-2000)," Blood, vol. 102, no. 4, pp. 1541-1547, 2003.

[23] Y. Morishima, T. Yabe, K. Matsuo et al., "Effects of HLA allele and killer immunoglobulin-like receptor ligand matching on clinical outcome in leukemia patients undergoing transplantation with T-cell-replete marrow from an unrelated donor," Biology of Blood and Marrow Transplantation, vol. 13, no. 3, pp. 315-328, 2007.

[24] R. Szydlo, J. M. Goldman, J. P. Klein et al., "Results of allogeneic bone marrow transplants for leukemia using donors other than HLA-identical siblings," Journal of Clinical Oncology, vol. 15, no. 5, pp. 1767-1777, 1997.

[25] T. Kawase, Y. Morishima, K. Matsuo et al., "High-risk HLA allele mismatch combinations responsible for severe acute graftversus-host disease and implication for its molecular mechanism," Blood, vol. 110, no. 7, pp. 2235-2241, 2007.

[26] L. Oevermann and R. Handgretinger, "New strategies for haploidentical transplantation," Pediatric Research, vol. 71, no. 4, part 2, pp. 418-426, 2012. 
[27] Y. Reisner, I. Ben-Bassat, D. Douer, A. Kaploon, E. Schwartz, and B. Ramot, "Demonstration of clonable alloreactive host T cells in a primate model for bone marrow transplantation," Proceedings of the National Academy of Sciences of the United States of America, vol. 83, no. 11, pp. 4012-4015, 1986.

[28] F. Aversa, A. Terenzi, A. Tabilio et al., "Full haplotype-mismatched hematopoietic stem-cell transplantation: a phase II study in patients with acute leukemia at high risk of relapse," Journal of Clinical Oncology, vol. 23, no. 15, pp. 3447-3454, 2005.

[29] C. Peters, S. Matthes-Martin, G. Fritsch et al., "Transplantation of highly purified peripheral blood $\mathrm{CD}^{+} 4^{+}$cells from HLAmismatched parental donors in 14 children: evaluation of early monitoring of engraftment," Leukemia, vol. 13, no. 12, pp. 20702078, 1999.

[30] Y. Reisner, N. Kapoor, D. Kirkpatrick et al., "Transplantation for severe combined immunodeficiency with HLA-A, B, D, DR incompatible parental marrow cells fractionated by soybean agglutinin and sheep red blood cells," Blood, vol. 61, no. 2, pp. 341-348, 1983.

[31] T. Klingebiel, J. Cornish, M. Labopin et al., "Results and factors influencing outcome after fully haploidentical hematopoietic stem cell transplantation in children with very high-risk acute lymphoblastic leukemia: impact of center size: an analysis on behalf of the Acute Leukemia and Pediatric Disease Working Parties of the European Blood and Marrow Transplant group," Blood, vol. 115, no. 17, pp. 3437-3446, 2010.

[32] N. A. Kernan, C. Bordignon, C. A. Keever et al., "Graft failures after T cell depleted marrow transplants for leukemia: clinical and in vitro characteristics," Transplantation Proceedings, vol. 19, no. 6, supplement 7, pp. 29-32, 1987.

[33] R. C. Barfield, M. Otto, J. Houston et al., "A one-step largescale method for T- and B-cell depletion of mobilized PBSC for allogeneic transplantation," Cytotherapy, vol. 6, no. 1, pp. 1-6, 2004.

[34] W. Leung, "Use of NK cell activity in cure by transplant," British Journal of Haematology, vol. 155, no. 1, pp. 14-29, 2011.

[35] C. Bottino, L. Moretta, D. Pende, M. Vitale, and A. Moretta, "Learning how to discriminate between friends and enemies, a lesson from natural killer cells," Molecular Immunology, vol. 41, no. 6-7, pp. 569-575, 2004.

[36] A. Moretta, F. Locatelli, and L. Moretta, "Human NK cells: from HLA class I-specific killer Ig-like receptors to the therapy of acute leukemias," Immunological Reviews, vol. 224, no. 1, pp. 5869, 2008.

[37] W. Leung, R. Iyengar, V. Turner et al., "Determinants of antileukemia effects of allogeneic NK cells," The Journal of Immunology, vol. 172, no. 1, pp. 644-650, 2004.

[38] L. Ruggeri, A. Mancusi, M. Capanni et al., "Donor natural killer cell allorecognition of missing self in haploidentical hematopoietic transplantation for acute myeloid leukemia: challenging its predictive value," Blood, vol. 110, no. 1, pp. 433-440, 2007.

[39] F. Locatelli, D. Pende, R. Maccario, M. C. Mingari, A. Moretta, and L. Moretta, "Haploidentical hemopoietic stem cell transplantation for the treatment of high-risk leukemias: how NK cells make the difference," Clinical Immunology, vol. 133, no. 2, pp. 171-178, 2009.

[40] R. Handgretinger, X. Chen, M. Pfeiffer et al., "Feasibility and outcome of reduced-intensity conditioning in haploidentical transplantation," Annals of the New York Academy of Sciences, vol. 1106, pp. 279-289, 2007.

[41] J. M. Goldman, R. P. Gale, M. M. Horowitz et al., "Bone marrow transplantation for chronic myelogenous leukemia in chronic phase: increased risk for relapse associated with T-cell depletion," Annals of Internal Medicine, vol. 108, no. 6, pp. 806-814, 1988.

[42] A. M. Marmont, M. M. Horowitz, R. P. Gale et al., "T-cell depletion of HLA-identical transplants in leukemia," Blood, vol. 78, no. 8, pp. 2120-2130, 1991.

[43] S. Chaleff, M. Otto, R. C. Barfield et al., "A large-scale method for the selective depletion of $\alpha \beta$ T lymphocytes from PBSC for allogeneic transplantation," Cytotherapy, vol. 9, no. 8, pp. 746754, 2007.

[44] E. Rådestad, H. Wikell, M. Engström et al., "Alpha/beta Tcell depleted grafts as an immunological booster to treat graft failure after hematopoietic stem cell transplantation with HLAmatched related and unrelated donors," Journal of Immunology Research, vol. 2014, Article ID 578741, 14 pages, 2014.

[45] M. Bonneville, R. L. O’Brien, and W. K. Born, “ $\gamma \delta$ T cell effector functions: a blend of innate programming and acquired plasticity," Nature Reviews Immunology, vol. 10, no. 7, pp. 467-478, 2010.

[46] S. Chiplunkar, S. Dhar, D. Wesch, and D. Kabelitz, " $\gamma \delta$ T cells in cancer immunotherapy: current status and future prospects," Immunotherapy, vol. 1, no. 4, pp. 663-678, 2009.

[47] K. T. Godder, P. J. Henslee-Downey, J. Mehta et al., "Long term disease-free survival in acute leukemia patients recovering with increased $\gamma \delta$ T cells after partially mismatched related donor bone marrow transplantation," Bone Marrow Transplantation, vol. 39, no. 12, pp. 751-757, 2007.

[48] J. L. M. Ferrara and P. Reddy, "Pathophysiology of graft-versushost disease," Seminars in Hematology, vol. 43, no. 1, pp. 3-10, 2006.

[49] A. Q. Gomes, D. S. Martins, and B. Silva-Santos, “Targeting $\gamma \delta$ $\mathrm{T}$ lymphocytes for cancer immunotherapy: from novel mechanistic insight to clinical application," Cancer Research, vol. 70, no. 24, pp. 10024-10027, 2010.

[50] W. Scheper, S. Van Dorp, S. Kersting et al., " $\gamma \delta$ T cells elicited by CMV reactivation after allo-SCT cross-recognize CMV and leukemia," Leukemia, vol. 27, no. 6, pp. 1328-1338, 2013.

[51] D. Balashov, A. Shcherbina, M. Maschan et al., "Single-center experience of unrelated and haploidentical stem cell transplantation with TCR $\alpha \beta$ and CD19 depletion in children with primary immunodeficiency syndromes," Biology of Blood and Marrow Transplantation, vol. 21, no. 11, pp. 1955-1962, 2015.

[52] C. Anasetti, D. Amos, P. G. Beatty et al., "Effect of HLA compatibility on engraftment of bone marrow transplants in patients with leukemia or lymphoma," The New England Journal of Medicine, vol. 320, no. 4, pp. 197-204, 1989.

[53] P. G. Beatty, R. A. Clift, E. M. Mickelson et al., "Marrow transplantation from related donors other than HLA-identical siblings," The New England Journal of Medicine, vol. 313, no. 13, pp. 765-771, 1985.

[54] "The host barrier in animal models of T-cell depleted allogeneic bone marrow transplantation. T-cell depletion in allogeneic bone marrow transplantation," in Serono Symposia Review, Y. Reisner, T. Lapidot, T. S. Singer, and E. Schwartz, Eds., 1988.

[55] P. J. Martin, “The role of donor lymphoid cells in allogeneic marrow engraftment," Bone Marrow Transplantation, vol. 6, no. 5, pp. 283-289, 1990.

[56] R. J. Soiffer, P. Mauch, N. J. Tarbell et al., "Total lymphoid irradiation to prevent graft rejection in recipients of HLA non-identical T cell-depleted allogeneic marrow," Bone Marrow Transplantation, vol. 7, no. 1, pp. 23-33, 1991. 
[57] F. Aversa, A. Tabilio, A. Velardi et al., "Treatment of high-risk acute leukemia with T-cell-depleted stem cells from related donors with one fully mismatched hla haplotype," The New England Journal of Medicine, vol. 339, no. 17, pp. 1186-1193, 1998.

[58] E. Bachar-Lustig, N. Rachamim, H.-W. Li, F. Lan, and Y. Reisner, "Megadose of T cell-depleted bone marrow overcomes MHC barriers in sublethally irradiated mice," Nature Medicine, vol. 1, no. 12, pp. 1268-1273, 1995.

[59] F. Aversa, A. Tabilio, A. Terenzi et al., "Successful engraftment of T-cell-depleted haploidentical 'three-loci' incompatible transplants in leukemia patients by addition of recombinant human granulocyte colony-stimulating factor-mobilized peripheral blood progenitor cells to bone marrow inoculum," Blood, vol. 84, no. 11, pp. 3948-3955, 1994.

[60] Y. Reisner and M. F. Martelli, "Bone marrow transplantation across HLA barriers by increasing the number of transplanted cells," Immunology Today, vol. 16, no. 9, pp. 437-440, 1995.

[61] E. Naparstek, M. Delukina, R. Or et al., "Engraftment of marrow allografts treated with Campath-1 monoclonal antibodies," Experimental Hematology, vol. 27, no. 7, pp. 1210-1218, 1999.

[62] J. L. M. Ferrara, R. Levy, and N. J. Chao, "Pathophysiologic mechanisms of acute graft-vs.-host disease," Biology of Blood and Marrow Transplantation, vol. 5, no. 6, pp. 347-356, 1999.

[63] N. A. Kernan, G. Bartsch, R. C. Ash et al., "Analysis of 462 transplantations from unrelated donors facilitated by the national marrow donor program," The New England Journal of Medicine, vol. 328, no. 9, pp. 593-602, 1993.

[64] L. Luznik, P. V. O’Donnell, H. J. Symons et al., "HLAhaploidentical bone marrow transplantation for hematologic malignancies using nonmyeloablative conditioning and highdose, posttransplantation cyclophosphamide," Biology of Blood and Marrow Transplantation, vol. 14, no. 6, pp. 641-650, 2008.

[65] H. Ogawa, K. Ikegame, S. Yoshihara et al., "Unmanipulated HLA 2-3 antigen-mismatched (haploidentical) stem cell transplantation using nonmyeloablative conditioning," Biology of Blood and Marrow Transplantation, vol. 12, no. 10, pp. 10731084, 2006.

[66] S. Kreiter, N. Winkelmann, P. M. Schneider et al., "Failure of sustained engraftment after non-myeloablative conditioning with low-dose TBI and T cell-reduced allogeneic peripheral stem cell transplantation," Bone Marrow Transplantation, vol. 28, no. 2, pp. 157-161, 2001.

[67] J. R. Passweg, S. Meyer-Monard, M. Gregor et al., "Nonmyeloablative stem cell transplantation: high stem cell dose will not compensate for $\mathrm{T}$ cell depletion in allogeneic nonmyeloablative stem cell transplantation," Bone Marrow Transplantation, vol. 30, no. 5, pp. 267-271, 2002.

[68] D. A. Rizzieri, L. P. Koh, G. D. Long et al., "Partially matched, nonmyeloablative allogeneic transplantation: clinical outcomes and immune reconstitution," Journal of Clinical Oncology, vol. 25, no. 6, pp. 690-697, 2007.

[69] P. V. O’Donnell, L. Luznik, R. J. Jones et al., "Nonmyeloablative bone marrow transplantation from partially HLA-mismatched related donors using posttransplantation cyclophosphamide," Biology of Blood and Marrow Transplantation, vol. 8, no. 7, pp. 377-386, 2002.

[70] H. Mayumi, K. Himeno, N. Tokuda, and K. Nomoto, "Druginduced tolerance to allografts in mice. VII. Optimal protocol and mechanism of cyclophosphamide-induced tolerance in an H-2 haplotype-identical strain combination," Transplantation Proceedings, vol. 18, no. 2, pp. 363-369, 1986.
[71] H. Mayumi, M. Umesue, and K. Nomoto, "Cyclophosphamideinduced immunological tolerance: an overview," Immunobiology, vol. 195, no. 2, pp. 129-139, 1996.

[72] L. Luznik, S. Jalla, L. W. Engstrom, R. Lannone, and E. J. Fuchs, "Durable engraftment of major histocompatibility complexincompatible cells after nonmyeloablative conditioning with fludarabine, low-dose total body irradiation, and posttransplantation cyclophosphamide," Blood, vol. 98, no. 12, pp. 3456-3464, 2001.

[73] L. Luznik, L. W. Engstrom, R. Iannone, and E. J. Fuchs, "Posttransplantation cyclophosphamide facilitates engraftment of major histocompatibility complex-identical allogeneic marrow in mice conditioned with low-dose total body irradiation," Biology of Blood and Marrow Transplantation, vol. 8, no. 3, pp. 131-138, 2002.

[74] R. J. Jones, J. P. Barber, M. S. Vala et al., "Assessment of aldehyde dehydrogenase in viable cells," Blood, vol. 85 , no. 10, pp. $2742-$ 2746, 1995.

[75] S. Sakaguchi, N. Sakaguchi, M. Asano, M. Itoh, and M. Toda, "Immunologic self-tolerance maintained by activated $\mathrm{T}$ cells expressing IL-2 receptor $\alpha$-chains (CD25): breakdown of a single mechanism of self-tolerance causes various autoimmune diseases," The Journal of Immunology, vol. 155, no. 3, pp. 11511164, 1995.

[76] Z. Fehérvari and S. Sakaguchi, "CD4 ${ }^{+}$Tregs and immune control," The Journal of Clinical Investigation, vol. 114, no. 9, pp. 1209-1217, 2004.

[77] C. I. Kingsley, M. Karim, A. R. Bushell, and K. J. Wood, "CD $25^{+} \mathrm{CD} 4^{+}$regulatory $\mathrm{T}$ cells prevent graft rejection: CTLA4- and IL-10-dependent immunoregulation of alloresponses," The Journal of Immunology, vol. 168, no. 3, pp. 1080-1086, 2002.

[78] J. Yu, X. Ren, F. Yan et al., "Alloreactive natural killer cells promote haploidentical hematopoietic stem cell transplantation by expansion of recipient-derived $\mathrm{CD} 4{ }^{+} \mathrm{CD} 25^{+}$regulatory $\mathrm{T}$ cells," Transplant International, vol. 24, no. 2, pp. 201-212, 2011.

[79] M. Edinger, P. Hoffmann, J. Ermann et al., "CD $4^{+} \mathrm{CD} 25^{+}$ regulatory $\mathrm{T}$ cells preserve graft-versus-tumor activity while inhibiting graft-versus-host disease after bone marrow transplantation," Nature Medicine, vol. 9, no. 9, pp. 1144-1150, 2003.

[80] A. Trenado, F. Charlotte, S. Fisson et al., "Recipient-type specific $\mathrm{CD} 4{ }^{+} \mathrm{CD} 25^{+}$regulatory $\mathrm{T}$ cells favor immune reconstitution and control graft-versus-host disease while maintaining graftversus-leukemia," The Journal of Clinical Investigation, vol. 112, no. 11, pp. 1688-1696, 2003.

[81] S. C. Jones, G. F. Murphy, and R. Korngold, "Post-hematopoietic cell transplantation control of graft-versus-host disease by donor $\mathrm{CD}^{+} 25^{+} \mathrm{T}$ cells to allow an effective graft-versusleukemia response," Biology of Blood and Marrow Transplantation, vol. 9, no. 4, pp. 243-256, 2003.

[82] K. Rezvani, S. Mielke, M. Ahmadzadeh et al., "High donor FOXP3-positive regulatory $\mathrm{T}$-cell $\left(\mathrm{T}_{\mathrm{reg}}\right)$ content is associated with a low risk of GVHD following HLA-matched allogeneic SCT," Blood, vol. 108, no. 4, pp. 1291-1297, 2006.

[83] V. H. Nguyen, S. Shashidhar, D. S. Chang et al., "The impact of regulatory $\mathrm{T}$ cells on $\mathrm{T}$-cell immunity following hematopoietic cell transplantation," Blood, vol. 111, no. 2, pp. 945-953, 2008.

[84] M. Di Ianni, F. Falzetti, A. Carotti et al., "Immunoselection and clinical use of $\mathrm{T}$ regulatory cells in HLA-haploidentical stem cell transplantation," Best Practice \& Research: Clinical Haematology, vol. 24, no. 3, pp. 459-466, 2011.

[85] A. M. Hanash and R. B. Levy, "Donor CD $4^{+} \mathrm{CD} 25^{+}$T cells promote engraftment and tolerance following MHC-mismatched 
hematopoietic cell transplantation," Blood, vol. 105, no. 4, pp. 1828-1836, 2005.

[86] P. Hoffmann and M. Edinger, " $\mathrm{CD} 4^{+} \mathrm{CD} 25^{+}$regulatory T cells and graft-versus-host disease," Seminars in Hematology, vol. 43, no. 1, pp. 62-69, 2006.

[87] M. Di Ianni, F. Falzetti, A. Carotti et al., “Tregs prevent GVHD and promote immune reconstitution in HLA-haploidentical transplantation," Blood, vol. 117, no. 14, pp. 3921-3928, 2011.

[88] M. Di Ianni, B. Del Papa, D. Cecchini et al., "Immunomagnetic isolation of $\mathrm{CD}^{+} \mathrm{CD}^{2} 5^{+} \mathrm{FoxP}^{+}$natural $\mathrm{T}$ regulatory lymphocytes for clinical applications," Clinical \& Experimental Immunology, vol. 156, no. 2, pp. 246-253, 2009.

[89] L. M. Williams and A. Y. Rudensky, "Maintenance of the Foxp3dependent developmental program in mature regulatory $\mathrm{T}$ cells requires continued expression of Foxp3," Nature Immunology, vol. 8, no. 3, pp. 277-284, 2007.

[90] D. J. Hartigan-O'Connor, C. Poon, E. Sinclair, and J. M. McCune, "Human CD4+ regulatory T cells express lower levels of the IL-7 receptor alpha chain (CD127), allowing consistent identification and sorting of live cells," Journal of Immunological Methods, vol. 319, no. 1-2, pp. 41-52, 2007.

[91] M. Battaglia, A. Stabilini, B. Migliavacca, J. Horejs-Hoeck, T. Kaupper, and M.-G. Roncarolo, "Rapamycin promotes expansion of functional $\mathrm{CD} 4{ }^{+} \mathrm{CD} 25^{+} \mathrm{FOXP} 3^{+}$regulatory $\mathrm{T}$ cells of both healthy subjects and type 1 diabetic patients," The Journal of Immunology, vol. 177, no. 12, pp. 8338-8347, 2006.

[92] J. Peccatori, A. Forcina, D. Clerici et al., "Sirolimus-based graftversus-host disease prophylaxis promotes the in vivo expansion of regulatory $\mathrm{T}$ cells and permits peripheral blood stem cell transplantation from haploidentical donors," Leukemia, vol. 29, no. 2, pp. 396-405, 2014.

[93] C. Anasetti, B. R. Logan, S. J. Lee et al., "Peripheral-blood stem cells versus bone marrow from unrelated donors," The New England Journal of Medicine, vol. 367, no. 16, pp. 1487-1496, 2012.

[94] O. Ringdén, M. Labopin, D. W. Beelen et al., "Bone marrow or peripheral blood stem cell transplantation from unrelated donors in adult patients with acute myeloid leukaemia, an Acute Leukaemia Working Party analysis in 2262 patients," Journal of Internal Medicine, vol. 272, no. 5, pp. 472-483, 2012.

[95] A. M. Raiola, A. Dominietto, A. Ghiso et al., "Unmanipulated haploidentical bone marrow transplantation and posttransplantation cyclophosphamide for hematologic malignancies after myeloablative conditioning," Biology of Blood and Marrow Transplantation, vol. 19, no. 1, pp. 117-122, 2013.

[96] P. D. Bartolomeo, S. Santarone, G. De Angelis et al., "Haploidentical, unmanipulated, G-CSF-primed bone marrow transplantation for patients with high-risk hematologic malignancies," Blood, vol. 121, no. 5, pp. 849-857, 2013.

[97] C. G. Kanakry, S. Ganguly, M. Zahurak et al., "Aldehyde dehydrogenase expression drives human regulatory $\mathrm{T}$ cell resistance to posttransplantation cyclophosphamide," Science Translational Medicine, vol. 5, no. 211, Article ID 211ra157, 2013. 


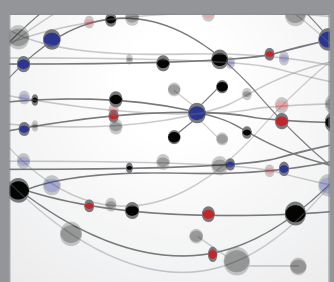

The Scientific World Journal
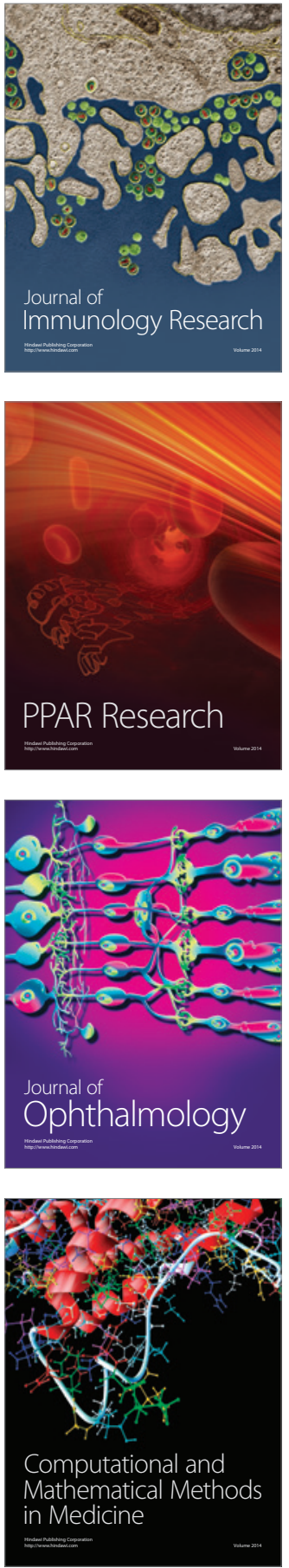

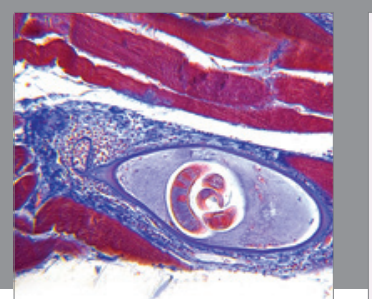

Gastroenterology Research and Practice

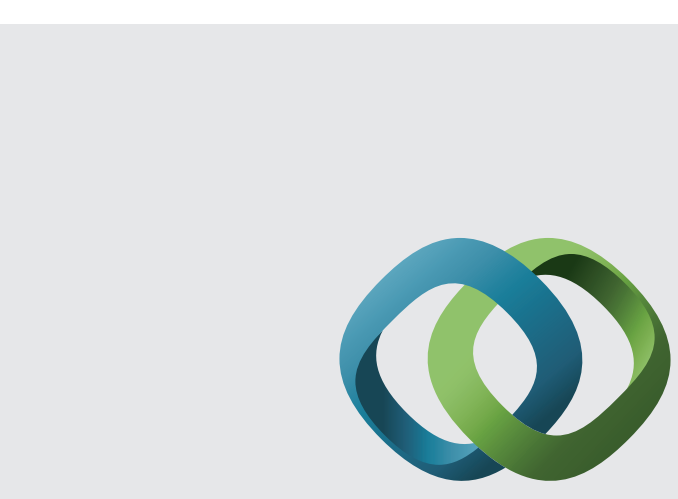

\section{Hindawi}

Submit your manuscripts at

http://www.hindawi.com
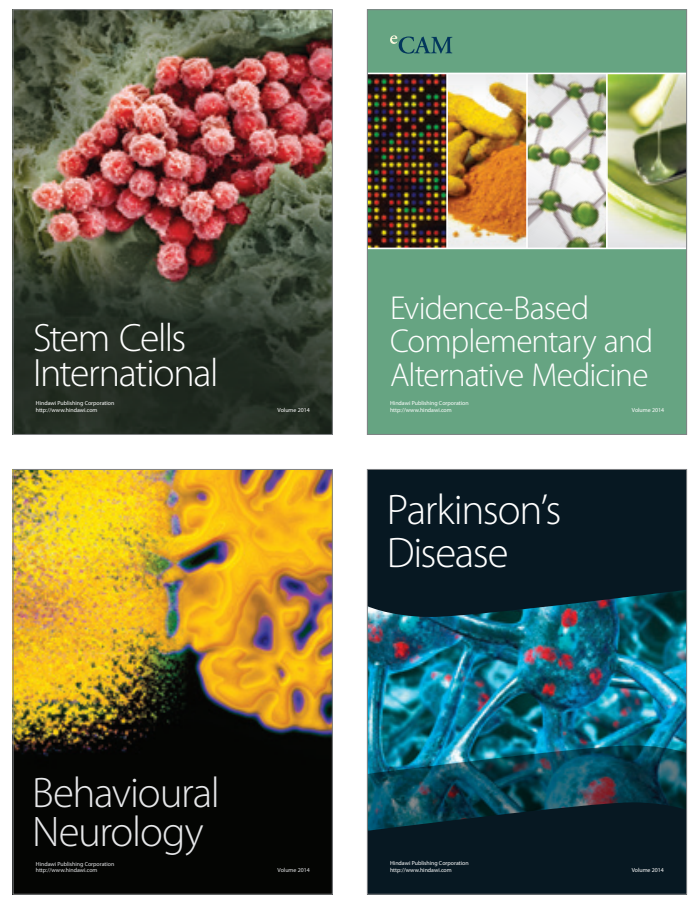
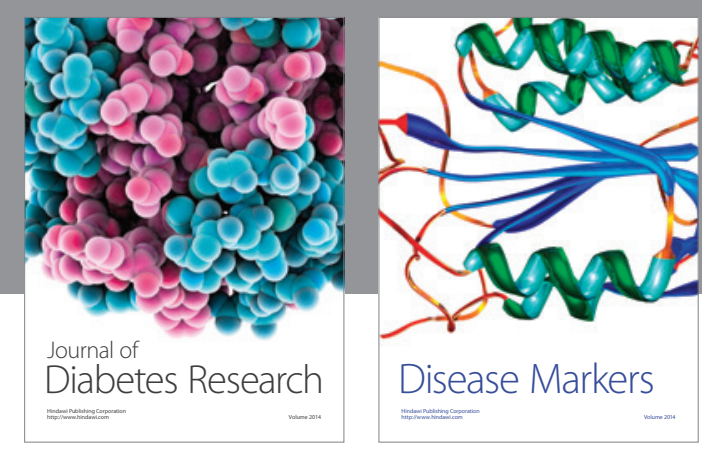

Disease Markers
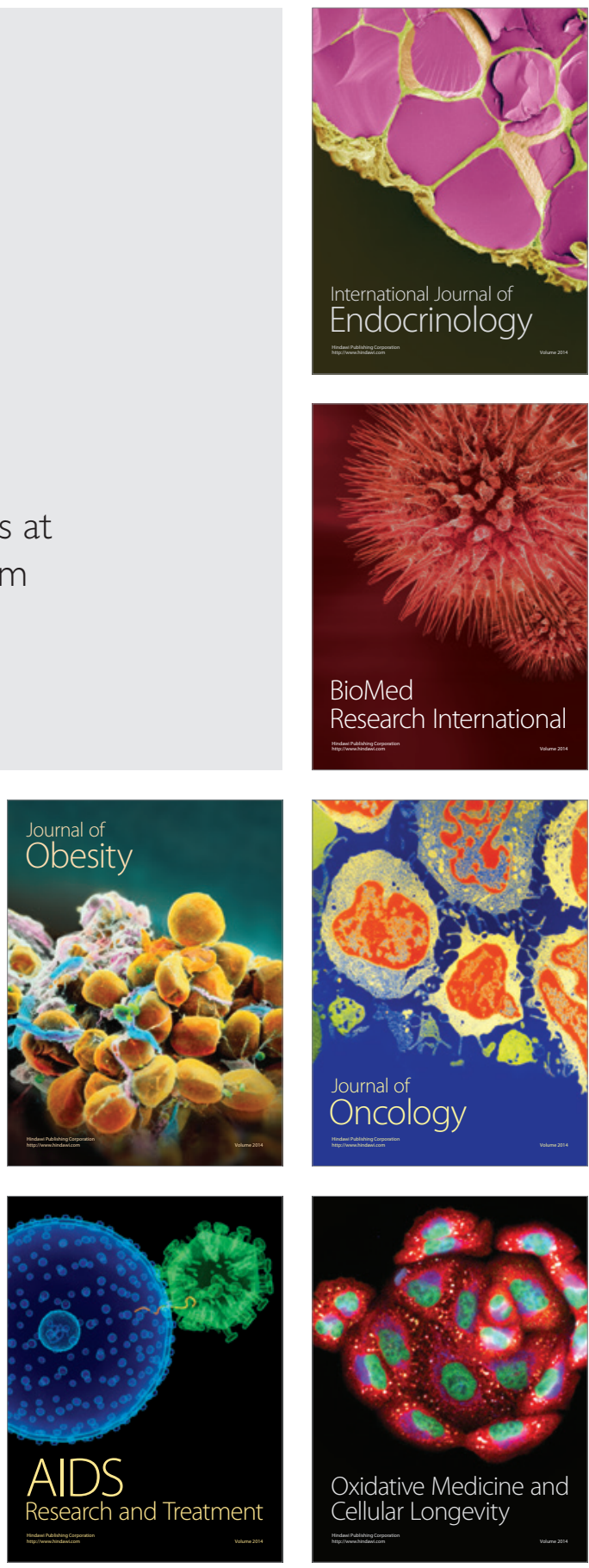